\title{
Microwave Radiation is a Versatile Technology for Tissue Processing
}

\author{
R. T. Giberson
}

Research and Development, Ted Pella, Inc., Redding, CA 96003

Having the benefit of hindsight allows us to look at where we came from in the science of microwave processing, where we are today and what the future might hold. The past helped to define the processing variables that are the important parameters in providing consistent results. Understanding and controlling the energy uniformity in the microwave cavity and evaluating the role of microwave-induced sample heating were the two major parameters early on [1]. To evaluate each the technology itself had to improve. Microwave devices had to evolve from fixed wattage instruments to true wattage devices. True wattage is simply the ability to regulate magnetron (source of microwaves) power output like a dimmer switch controls a light. Secondly, a tool had to be developed to provide energy uniformity in the microwave cavity.

Giberson et al. (2003) demonstrated the benefits of true wattage and energy uniformity when it came to processing unfixed tissue through all the steps of sample preparation for electron microscopy. They reported that turnaround times could be reduced over 20 -fold from conventional methods in conjunction with excellent tissue ultrastructure. The same methodology was applied to immunolabeling techniques in the neurosciences with the same results. Significant time savings ( $>20$-fold) were reported in conjunction with excellent labeling [3]. In both papers the creation of energy uniformity was cited as important to the processing results [2-3]. Table 1 is a demonstration of that uniformity over a wide range of true wattage output. The parameter left to define was the role of sample temperature increase during microwave-assisted processing.

Tinling et al. (2004) described a microwave system where sample temperature control could be provided from outside the microwave cavity. They described microwave-assisted decalcification using true wattage at a constant sample temperature. The results of their work provided direct experimental evidence for a microwave promoted process. Shortly after Galvez et al. (2006), using the same experimental design as Tinling et al. (2004), reported that fresh tissue could be formaldehyde fixed in the microwave in as little as 20 minutes. Figure 1 is the ultrastructural evidence demonstrating the efficacy of microwave-assisted rapid formaldehyde fixation.

The versatility of microwave-assisted processing has been chronicled over the last 8 years for a number of tissue processing applications [2-5]. That success has been dependent on creating uniformity in the microwave cavity and providing sample temperature control through both the application of true wattage and external sample temperature control [2-5].

\section{References}

[1] R.T. Giberson et al., J Vet Diagn Invest (1997) 9.

[2] R.T. Giberson et al., Ultrastruct Pathol (2003) 27.

[3] T. E. Munoz et al., J Neurosci Methods (2004) 137.

[4] S. P. Tinling et al., J. Microsc (2004) 215.

[5] J. J. Galvez et al., The J Histotechnol (2006) 29. 
Table 1. Energy uniformity supplied by the ColdSpot [2-3] at different true wattages. Temperature data from 13 trials and 80 seconds of continuous microwave irradiation.
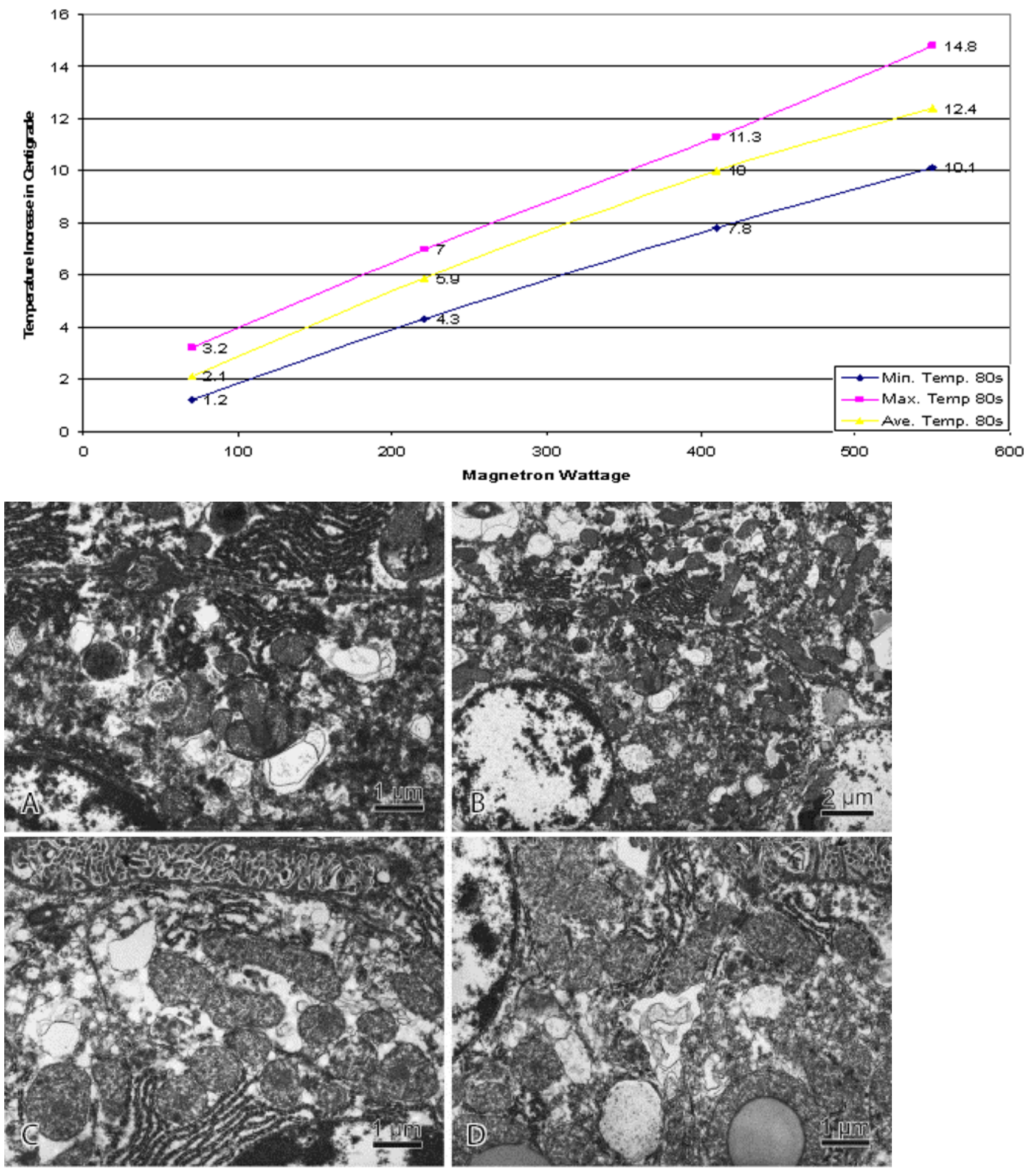

Fig. 1. Ultrastructural comparison of ovine liver fixed for 24 hours in $10 \%$ neutral buffered formalin (A-B) to that of a 20 minute microwave-assisted fixation (C-D) [5]. The fine detail in the microwave fixed tissue is evident when compared to the conventionally fixed tissue. The speed and quality of microwave methods is evident. 